\title{
Investigation of Causal Relationship Between Touch Sensations of Robots and Personality Impressions by Path Analysis
}

\author{
Yuki Yamashita $^{1} \cdot$ Hisashi Ishihara $^{1}$ (D) Takashi Ikeda $^{2} \cdot$ Minoru Asada $^{1}$
}

Accepted: 16 May 2018 / Published online: 7 June 2018

(c) The Author(s) 2018

\begin{abstract}
Physical human-robot interaction plays an important role in social robotics, and touch is one of the key factors that influence a human's impression of robots. However, very few studies have explored different conditions pertaining to such interactions, and therefore, only few systematic results have been reported. As the first step toward addressing this issue, we studied the types of impressions of a robot's personality that humans may experience when they touch a soft part of the robot. In the study, the left forearm of a child-like android robot named "Affetto" was exposed; the original forearm was made of silicone rubber and can be replaced with any one of three other forearms that provide different sensations of hardness upon touching. Participants were asked to touch the robot's forearm and answer evaluation questionnaires on 19 touch sensations and 46 personality impressions for each of the four conditions associated with the different forearms. Four impression factors for touch sensations and three for personality impressions were extracted from the evaluation scores by the factor analysis method. The causal relationships between these factors were analyzed by the path analysis method. Several significant causal relationships were found, for example, between preferable touch sensations and likable personality impressions. The study results are expected to help in the design of the personality impressions of robots by facilitating a more systematic design of touch sensations. Furthermore, the results of an analysis of variance suggested that the most preferable touch sensation is possibly related to the robot's appearance and that designers of social robots should understand the complex touch sensation properties of humans.
\end{abstract}

Keywords Touch sensation · Personality impression · Factor analysis · Path analysis · Soft covering

\section{Introduction}

The quality of social interaction depends on various nonsocial factors just as the quality of verbal communication

This research is supported by Grants-in-Aid for Specially Promoted Research (No. JK24000012) and for Young Scientists (B) (No. JK15K18006) and by the Center of Innovation Program of MEXT and JST.

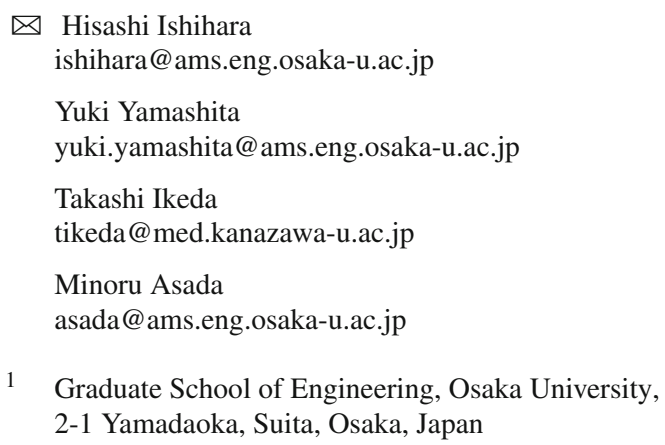

depends on many non-verbal parameters [1]. In social robotics, physical human-robot interaction typically improves the social interaction between humans and robots [2]. One of the important issues in the design of communication robots is the selection of the covering material with appropriate touch sensations to improve the personality impressions of robots. The appearance of robots affects their personality impressions [3-6]. However, for humans, personality impressions may be influenced by not only visual perception but also some other modalities. Volume and tone are major components that inform the listener about the emotional state of the speaker [7]. Tactile sensations produced by touching products affect the quality impressions and attractiveness of the products. Therefore, it seems reasonable to consider that the touch of robots also influences their personality impressions.

2 Research Center for Child Mental Development, Kanazawa University, 13-1 Takaramachi, Kanazawa, Ishikawa, Japan 
(a) Factor Analysis

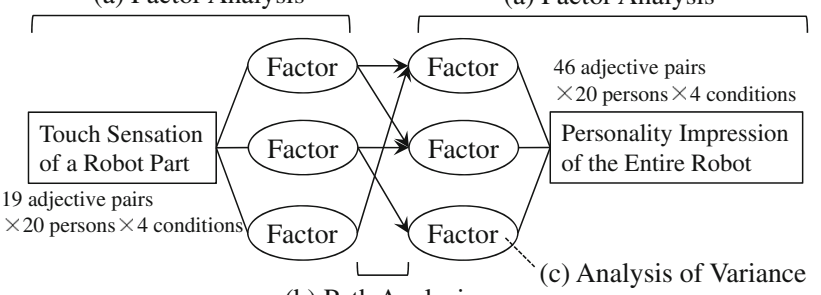

(b) Path Analysis (c) Analysis of Variance

(ANOVA)
Fig. 1 Research overview of a potential causal relationship between the touch sensations of a robot part and the personality impressions of the entire robot (modified from [8]). Factor analysis was carried out to identify abstract factors from evaluation scores obtained by assessing questionnaire results. Path analysis was carried out, which revealed significant causal relationships between these factors. Then, an analysis of variance of the identified factors was carried out to investigate the differences in the evaluations among the different forearms

Several studies have attempted to realize touch-based interactions between humans and robots [9-12], and designers have selected comfortable covering materials for robots to influence the mental states of humans through the sensation of touch [13-17]. However, the design of the touch sensation of robots to improve their intended personality impressions is a challenge because the types of touch sensations that affect the personality impressions and the manner in which these sensations produce these effects have not been systematically investigated.

The touch sensations of robot skins [18], personality impressions of hugging dolls [19], and both the touch sensations and personality impressions of robot hands [20] were evaluated under different conditions, but the manner in which touch sensations affect personality impressions has not been reported so far. Therefore, touch sensations of robots have been determined intuitively and empirically and tuned in a try-and-error manner by designers. This inefficient situation could be improved if the causal relationship from touch sensations of robots to personality impressions of them is revealed. In other words, the designers can determine appropriate touch sensations of robots that provide their intended personality impressions systematically and efficiently.

To systematically understand the causal relationships between touch sensations and personality impressions, we have examined the perceived impressions of a robot by asking human participants to answer questionnaires containing a large number of questions [8]. In our previous study, we found significant causal relationships between tactile impression factors and personality impression factors with four types of silicone rubber forearms attached to a stationary robot. In this study, we analyzed the causal relationships in more detail to investigate how each forearm was evaluated by the participants. Figure 1 shows an overview of our study. Participants were instructed to touch and grab the silicone rubber forearm of a stationary robot using their right hand and then answer the evaluation questionnaires on touch sensations (19 items) and personality impressions (46 items) under each of the four conditions associated with different forearms that provide different sensations of hardness upon touching. A factor analysis of the evaluation scores was carried out to identify abstract impression factors for touch sensations and personality impressions [8]. A path analysis was then carried out, which revealed significant causal relationships between tactile impression factors and personality impression factors [8]. Finally, an analysis of variance (ANOVA) was carried out to investigate the difference in the evaluations by the participants among the different forearms.

\section{Method}

\subsection{Participants}

The participants were 20 healthy Japanese adults, including 10 males (mean age $=21.9, S D=2.3$ ) and 10 females (mean age $=22.9, S D=1.2$ ). Of these, 17 participants had no experience of contact with humanoid robots until the experiment, and 11 participants had no knowledge of humanoid robots. Two participants had contact with infants in the past 5 years. All participants provided written informed consent approved by the Ethics Committee of Graduate School of Engineering, Osaka University.

\subsection{Robot}

A child-like android robot named Affetto [21] was set on a desk in front of a chair, as shown in Fig. 2. The robot had a head and upper body, which were covered with a cloth or gloves; only its face and left forearm were exposed. The joints of the robot were physically fixed to maintain a particular posture and its left hand was held out to the participants so that the joint movements did not affect its impressions on the participants. A partition was placed between the robot and participants so that the robot could be hidden during forearm exchanges and could be shown to the participants only during the experiment.

Four different types of left forearms-A, B, C, and Dwith identical sizes and appearances were prepared, and they were set (one at a time) between the left elbow and left hand of the robot under each experimental condition. Figure 3 shows the structural overview of the left forearm. Overall, the forearms were cylindrical with a diameter of $40 \mathrm{~mm}$ and a length of $105 \mathrm{~mm}$. The main material of the forearm was cured platinum silicone rubber (Dragon Skin FX-Pro, Smooth-On Inc.), and its center was supported by a metal rod with a diameter of $8 \mathrm{~mm}$. The outer surface of the silicone rubber was wrapped with a thin $(7 \mu \mathrm{m})$ polyurethane film (Airwall 


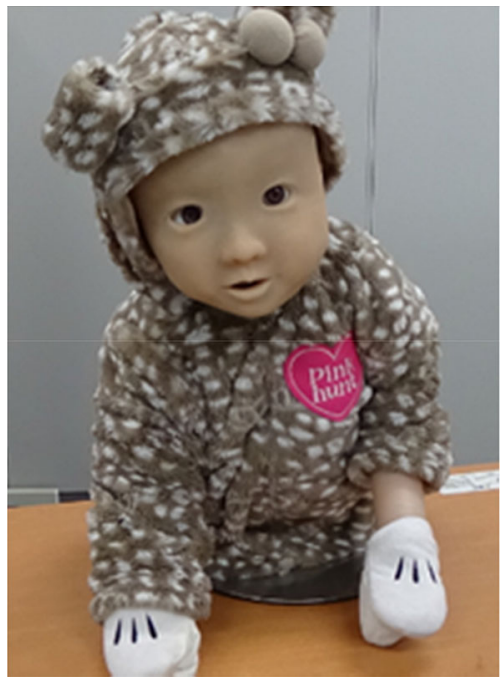

Fig. 2 Appearance of the robot with exposed left forearm. Reproduced with permission from Yamashita et al. [8]

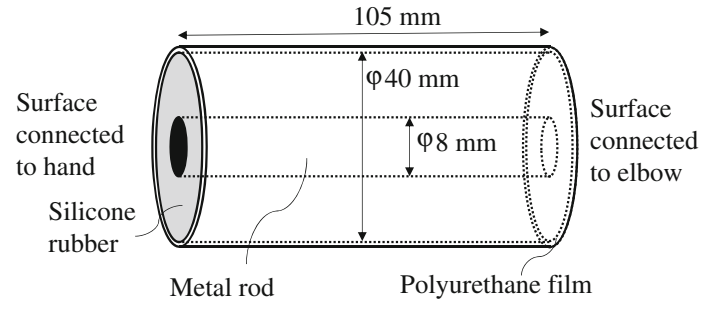

Fig. 3 Structural overview of the left forearm. Modified from Yamashita et al. [8]

UV, Kyowa Ltd.) to ensure constant surface friction along the forearm.

Different amounts of two types of additives were mixed with the silicone rubber to provide different touch sensations to the forearms. One of the additives was a plasticizer (Silicone Thinner, Smooth-On Inc.), which reduces hardness, whereas the other was a thickener (Slacker, Smooth-On Inc.), which increases viscosity while reducing hardness. The additive contents were adjusted so that the hardness decreased from forearm $\mathrm{A}$ to forearm $\mathrm{D}^{1}$, and so that forearms $\mathrm{C}$ and $\mathrm{D}$ had higher viscosity than forearms $\mathrm{A}$ and $\mathrm{B}$. The different percentages of the plasticizer and thickener used for preparing the forearms are listed in Table 1.

\footnotetext{
1 The elasticity, which was measured using a durometer (ASKER Durometer Type FP, Kobunshi Keiki Co., Ltd.) was the highest for fore$\operatorname{arm~A,~followed~by~forearms~B,~C,~and~D~in~this~order.~The~measured~}$ elasticities of forearms B, C, and D were similar to those of the center of the back of the co-contracted forearm of males, relaxed forearm of males (or forceful forearm of females), and relaxed forearm of females, respectively.
}

Table 1 Amounts of two types of additives used for preparing the four forearms

\begin{tabular}{lll}
\hline Forearm & Plasticizer $(\%)$ & Thickener $(\%)$ \\
\hline A & 10 & 0 \\
B & 50 & 0 \\
C & 10 & 30 \\
D & 20 & 30 \\
\hline
\end{tabular}

Note that the volume ratios of additives are relative to the volume of the silicone rubber before the additives were added

\subsection{Questionnaires}

The semantic differential (SD) method [22] was used to measure the touch sensations and personality impressions. Two sets of a touch sensation questionnaire (TSQ) and personality impression questionnaire (PIQ), each with lists of several pairs of opposite Japanese adjectives, were provided to the participants. They were instructed to choose their responses on a seven-point scale between opposite adjectives, e.g., "Soft or Hard" for the TSQ and "Active or Passive" for the PIQ.

Table 2 summarizes the 19 adjective pairs used in the TSQ. Most of these pairs were selected from previous studies on touch sensations of artificial skins with eight adjective pairs [18] and touch sensations of robot hands with 10 adjective pairs [20]. Table 3 summarizes the 46 adjective pairs used in the PIQ. Most of these pairs were derived or selected from previous studies on personality impressions of robot hands with 12 pairs [20], impressions of hug dolls with 12 adjective pairs [19], quantification of impressions of humanoid robots with 33 adjective pairs, and meta-analysis of SD adjective pairs for personality impressions [23]. Thus, our adjective pairs were prepared such that we could investigate touch sensations and personality impressions thoroughly. These adjective pairs were translated into English by a professional translator for use in this manuscript.

\subsection{Procedure}

The experimental procedures were divided into three sessions. In the first one, the participants were instructed on the manner in which the robot was to be evaluated. In the second one, they practiced evaluating their own forearms in the instructed manner. In the third one, they evaluated the robot by touching its forearm and then answering the questionnaires.

In the first instruction session, a movie describing the manner of touching the robot and the two questionnaires (TSQ and PIQ) were shown to the participants. The movie showed a demonstrator pinching one of the forearms of the robot with his thumb and forefinger, touching the forearm with the pads 
Table 2 Adjective pairs used in the touch sensation questionnaire (TSQ). Reproduced with permission from Yamashita et al. [8]
Table 3 Adjective pairs used in the personality impression questionnaire (PIQ). Reproduced with permission from Yamashita et al. [8]

\begin{tabular}{llrlll}
\hline$\#$ & Adjective pair & $\#$ & Adjective pair & $\#$ & Adjective pair \\
\hline 1 & Flabby/supple & 8 & Light/heavy & 14 & Coarse/fine \\
2 & Complex/simple & 9 & Comfortable/uncomfortable & 15 & Good/bad \\
3 & Dry/moist & 10 & Tense/relaxed & 16 & Pleasant/unpleasant \\
4 & Bad-feeling/good-feeling & 11 & Blunt/sharp & 17 & Elastic/rigid \\
5 & Soft/hard & 12 & Slippery/sticky & 18 & Smooth/rough \\
6 & Large/small & 13 & Slim/plump & 19 & Rounded/angular \\
7 & Desirable/undesirable & & & &
\end{tabular}

\begin{tabular}{|c|c|c|c|}
\hline \# & Adjective pair & \# & Adjective pair \\
\hline 1 & Amiable/odious & 24 & Masculine/feminine \\
\hline 2 & Humanlike/machinelike & 25 & Agreeable/disagreeable \\
\hline 3 & Tiresome/endlessly entertaining & 26 & Safe/dangerous \\
\hline 4 & Active/passive & 27 & Wise/foolish \\
\hline 5 & Earnest/insincere & 28 & Good/bad \\
\hline 6 & Pain-sensitive/pain-insensitive & 29 & Quiet/noisy \\
\hline 7 & Kind/unkind & 30 & Friendly/unfriendly \\
\hline 8 & Amusing/boring & 31 & Merry/objectionable \\
\hline 9 & Lively/unlively & 32 & Mild-mannered/strict \\
\hline 10 & Talkative/reticent & 33 & Jovial/gloomy \\
\hline 11 & Soothing/not soothing & 34 & Convivial/stiff-mannered \\
\hline 12 & Vigorous/lifeless & 35 & Extroverted/introverted \\
\hline 13 & Considerable/self-centered & 36 & Robust/feeble \\
\hline 14 & Reassuring/unnerving & 37 & Laid-back/busy \\
\hline 15 & Young/old & 38 & Approachable/unapproachable \\
\hline 16 & Reliable/unreliable & 39 & Spritely/fatigued \\
\hline 17 & Bright/dismal & 40 & Comfortable/uncomfortable \\
\hline 18 & Docile/obstinate & 41 & Clean/dirty \\
\hline 19 & Pleasant/unpleasant & 42 & Adorable/weird \\
\hline 20 & Brave/cowardly & 43 & Sturdy/fragile \\
\hline 21 & Calm/restless & 44 & Neat/slovenly \\
\hline 22 & Desirable/undesirable & 45 & Confident/timid \\
\hline 23 & Warmhearted/cold-hearted & 46 & Strong/weak \\
\hline
\end{tabular}

of his fingers, and holding the forearm with his hand several times. The participants were told to look at the forearm and touch it with their dominant hand as shown in the movie. In the second session, for training purposes, the participants were told to touch their own forearm with their dominant hand and answer the TSQ and PIQ. This session was conducted to check whether the participants had understood the instructions regarding the manner of touching the forearm and to allow the participants to get acquainted with the questionnaires. The third evaluation session was divided into four subsessions in which the participants evaluated each of the four forearms attached to the robot and the entire robot. In each subsession, the participants were instructed to touch the forearm of the robot for arbitrary time durations and then answer the TSQ. After answering it, they were told to touch the forearm again and then answer the PIQ. This subsession took approximately $10 \mathrm{~min}$. Between subsessions, the robot was hidden from the participants by the partition, and the robot's attached forearm was changed; then, the robot was shown to the participants again. The forearm replacement was completed in $1 \mathrm{~min}$. The order in which each forearm was showed and the order of the adjective pairs in the questionnaires were shuffled for each participant. The entire series of sessions was completed in an hour. 


\subsection{Data Processing}

An exploratory factor analysis was carried out to identify several underlying factors, each of which was statistically reflected by several observed variables or evaluation scores of the adjective pairs. The maximum-likelihood estimation and varimax rotation were chosen as the factor extraction and rotation methods, respectively. The number of factors chosen according to the scree test was investigated by the Bayesian information criterion (BIC) and the root mean square error of approximation (RMSEA).

Path analysis was carried out to determine significant causal relationships between the found factors. Here, we assumed a multivariate multiple regression model whose independent variables were the touch sensation factors and the dependent variables were the personality impression factors. The maximum-likelihood estimation was used for estimating the model parameters. The $\mathrm{R}$ language (version 3.2.2) [24] was used for the above analyses.

\section{Results}

\subsection{Factor Analysis}

To detect the model structure, the number of dimensions should be reduced. We carried out factor analyses of each questionnaire by using the number of factors determined by inspecting the scree plot of eigenvalues. Four touch feeling factors $(\mathrm{BIC}=-292.2, \mathrm{RMSEA}=0.094)$ and three personality impression factors $(\mathrm{BIC}=-2774.5$, RMSEA $=$ $0.009)$ were extracted from the evaluation scores of each questionnaire. Tables 4 and 5 list the factor matrices for the touch sensation factors and personality impression factors, respectively. Based on the adjectives with high loadings for each factor, we named factor 1 as "Preference," factor 2 as "Resilience," factor 3 as "Smoothness," and factor 4 as "Naturalness" for touch sensations. We named factor 1 as "Likability," factor 2 as "Capability," and factor 3 as "Vitality" for personality impressions.

\subsection{Path Analysis}

We carried out path analysis to investigate how tactile feelings affect the impressions of a humanoid robot. The seven
Table 4 Factor matrix for the touch sensation factors. Reproduced with permission from Yamashita et al. [8]

\begin{tabular}{|c|c|c|c|c|}
\hline \multirow[t]{3}{*}{ Adjective } & \multicolumn{4}{|l|}{ Factor } \\
\hline & 1 & 2 & 3 & 4 \\
\hline & Preference & Resilience & Smoothness & Naturalness \\
\hline Good-feeling & $(0.97)$ & & & \\
\hline Pleasant & $(0.97)$ & & & \\
\hline Desirable & $(0.85)$ & & & \\
\hline Good & $(0.79)$ & & & \\
\hline Comfortable & 0.41 & & & \\
\hline Slippery & 0.32 & & & \\
\hline Supple & & $(0.91)$ & & \\
\hline Tense & & $(0.88)$ & & \\
\hline Rigid & & $(0.76)$ & & \\
\hline Hard & & $(0.69)$ & -0.33 & \\
\hline Heavy & -0.32 & 0.44 & & \\
\hline Large & & 0.32 & & \\
\hline Smooth & & & $(0.85)$ & \\
\hline Fine & & & $(0.75)$ & \\
\hline Simple & & & 0.42 & \\
\hline Rounded & & & 0.31 & $(0.59)$ \\
\hline Moist & & & & $(.52)$ \\
\hline Blunt & & -0.30 & & $(0.52)$ \\
\hline Plump & & & & 0.38 \\
\hline Accumulated variance (\%) & 36 & 67 & 86 & 100 \\
\hline
\end{tabular}

Loadings higher than the absolute value of 0.50 are shown in parentheses, and those lower than the absolute value of 0.30 are extracted 
Table 5 Factor matrix for the personality impression factors. Reproduced with permission from Yamashita et al. [8]

\begin{tabular}{|c|c|c|c|c|c|c|c|}
\hline \multirow[t]{3}{*}{ Adjective } & \multicolumn{3}{|l|}{$\underline{\text { Factor }}$} & \multirow[t]{3}{*}{ Adjective } & \multicolumn{3}{|l|}{ Factor } \\
\hline & 1 & 2 & 3 & & 1 & 2 & 3 \\
\hline & Likability & Capability & Vitality & & Likability & Capability & Vitality \\
\hline Desirable & $(0.99)$ & & & Masculine & & $(0.83)$ & \\
\hline Agreeable & $(0.93)$ & & & Brave & & $(0.79)$ & 0.33 \\
\hline Pleasant & $(0.90)$ & & & Confident & & $(0.78)$ & 0.33 \\
\hline Comfortable & $(0.90)$ & & & Neat & 0.39 & $(0.66)$ & \\
\hline Good & $(0.90)$ & & & Obstinate & -0.30 & (.66) & \\
\hline Friendly & $(0.79)$ & & & Busy & & $(0.65)$ & \\
\hline Soothing & $(0.78)$ & & & Strict & -0.31 & $(0.64)$ & \\
\hline Approachable & $(0.75)$ & & & Extrovert & & $(0.56)$ & $(0.60)$ \\
\hline Merry & $(0.73)$ & & & Stiff-mannered & & $(0.59)$ & \\
\hline Adorable & $(0.73)$ & & & Pain-sensitive & & $(0.51)$ & -0.41 \\
\hline Endlessly entertaining & $(0.65)$ & & & Talkative & & & $(0.82)$ \\
\hline Reassuring & $(0.64)$ & & & Noisy & & & $(0.81)$ \\
\hline Amiable & $(0.63)$ & -0.33 & & Jovial & & & $(0.79)$ \\
\hline Humanlike & $(0.61)$ & & & Bright & 0.31 & & $(0.79)$ \\
\hline Clean & $(0.52)$ & & & Active & & 0.48 & $(0.66)$ \\
\hline Considerable & $(0.52)$ & & & Lively & & 0.32 & $(0.66)$ \\
\hline Robust & & $(0.92)$ & & Spritely & 0.32 & & $(0.65)$ \\
\hline Strong & & $(0.91)$ & & Restless & -0.49 & & $(0.53)$ \\
\hline Reliable & & $(0.87)$ & & Accumulated variance $(\%)$ & 44 & 76 & 99 \\
\hline
\end{tabular}

Loadings higher than the absolute value of 0.50 are shown in parentheses and those lower than the absolute value of 0.30 are extracted

variables considered were the factor scores calculated from the factor analyses. A satisfactory goodness-of-fit index (RMSEA $<0.001$ ) for a full model including all of the possible paths was achieved.

Figure 4 shows the path diagram in which standardized partial regression coefficients represent the relationships between touch sensation factors and personality impression factors. The path thickness represents the magnitude of the

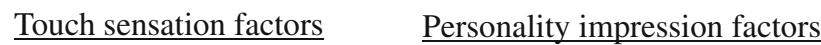

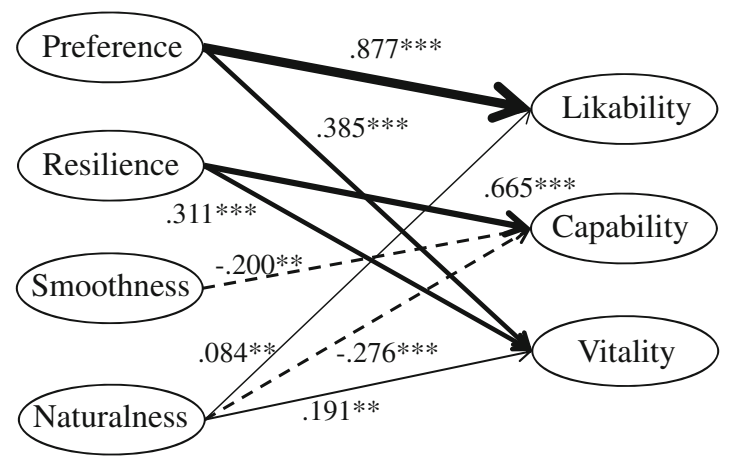

Fig. 4 Path diagram in which standardized coefficients represent the relationships between the touch sensation factors and personality impression factors [8]. The insignificant paths were removed. $* * p<$ $0.01, * * * p<0.001$ coefficient. Solid and dotted lines represent positive and negative relationships, respectively.

We found that the Likability personality impression was highly and positively affected by the Preference touch sensation $(\beta=0.877)$; the Capability personality impression was positively influenced by the Resilience touch sensation $(\beta=0.665)$; and the Vitality personality impression was positively affected by the Preference and Resilience touch sensations ( $\beta=0.385$ and 0.311$)$. Additionally, the Smoothness touch sensation affected the Capability personality impression negatively but weakly $(\beta=-0.200)$, and the Naturalness touch sensation affected all three personality factors weakly $(\beta=0.084,-0.276$, and 0.191$)$.

\subsection{ANOVA of Factor Scores}

To evaluate the differences among the four types of forearms, we carried out seven ANOVAs for the three touch sensation factors and four personality impression factors. For each analysis, we used a one-way repeated-measures ANOVA and a multiple comparisons test using Shaffer's modified Bonferroni procedure. All statistical thresholds were set at $p<0.05$.

Figure 5 shows the comparisons of the factor scores related to each touch sensation factor among the forearms. The main effect of the Preference factor was significant $[F(3,57)=$ 
Fig. 5 Comparisons of factor scores related to each touch sensation factor among the forearms. Each error bar shows the standard error of the mean. $\dagger p<0.1, * p<0.05, * * p<$ $0.01, * * * p<0.001$. a Preference factor, b resilience factor, c smoothness factor, $\mathbf{d}$ naturalness factor

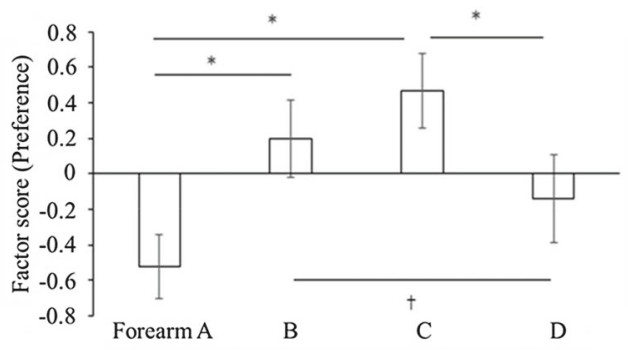

(a) Preference factor

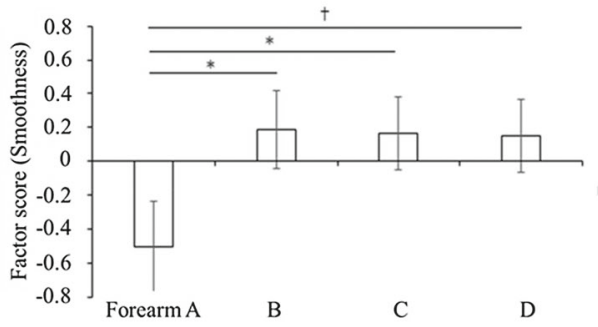

(c) Smoothness factor

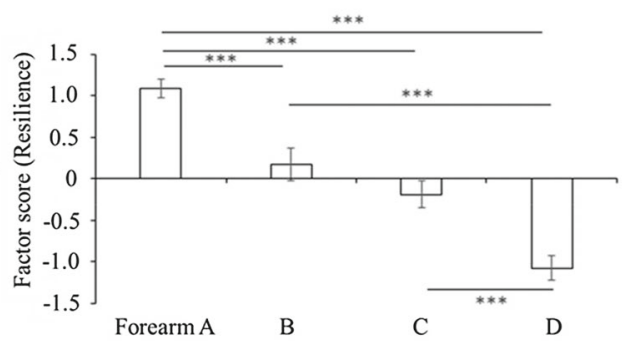

(b) Resilience factor

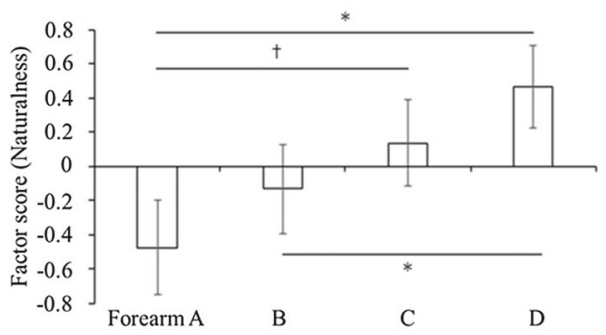

(d) Naturalness factor
$6.720, p<0.001]$. Forearms $\mathrm{B}$ and $\mathrm{C}$ had higher scores than forearms $\mathrm{A}$ and $\mathrm{D}$, which means that forearms $\mathrm{B}$ and $\mathrm{C}$ were evaluated to be more preferable (good-feeling and pleasant; Fig. 5a). The main effect of the Resilience factor was significant $[F(3,57)=41.87, p<0.001]$. Forearm A had the highest score related to the Resilience factor, whereas forearm $\mathrm{D}$ had the lowest score, which means that forearm A was evaluated to be the most resilient (supple and tense; Fig. 5b). The main effect of the Smoothness factor was significant $[F(3,57)=$ 5.112, $p=0.003]$. Forearm A had the lowest score related to the Smoothness factor, which means that forearm A was evaluated to be the smoothest (rough and coarse; Fig. 5c). The main effect of the Naturalness factor was significant $[F(3$, $57)=5.235, p=0.003]$. Forearm $\mathrm{D}$ had the highest score related to the Naturalness factor, whereas forearm A had the lowest score, which means that forearm D was evaluated to be the most natural (rounded and moist; Fig. 5d).

Figure 6 shows the comparisons of the factor scores related to each personality impression factor among the forearms. The main effect of the Likability factor was significant $[F(3$, $57)=5.469, p=0.002]$. Forearm $\mathrm{C}$ had the highest scores related to the Likability factor whereas forearm $\mathrm{A}$ had the lowest scores, which means that forearm $\mathrm{C}$ was evaluated to be more likable (desirable and agreeable; Fig. 6a). The main effect of the Capability factor was significant $[F(3,57)$ $=31.10, p<0.001]$. Forearm A had the highest score related to the Capability factor whereas forearm D had the lowest score, which means that forearm A was evaluated to be the most capable (robust and strong; Fig. 6b). However, the main effect of the Vitality factor was not significant $[F(3,57)=$ $1.841, p=0.150]$. No significant difference was found in the scores related to the Vitality factor as shown in Fig. 6c.

\section{Discussion}

Several significant causal relationships between the touch sensation factors and personality impression factors were identified by the path analysis in accordance with our hypothesis. In other words, even when a robot had a fixed appearance, its personality impression could be modified by touch sensations. Thus, this study systematically showed how different touch sensation factors affected different personality impression factors.

First, we found that the Likability personality impression was improved if the robot provided preferable and natural touch sensations to the participants. This supports the conventional idea of covering communication robots with good-feeling and lifelike materials such as fur [1416] and soft silicone rubber [20,25]. In particular, preferable touch sensations strongly affect the Likability personality impression, and this emphasizes the importance of designing preferable touch feelings to build likable robots. We consider that the strong causal relationship between preferable touch sensation and likable personality is the result of a type of halo effect, known as human cognitive bias, and is defined as the influence of a global evaluation on the evaluations of individual attributes of a person [26]. The Likability personality impression has been considered as one of the important properties for communication robots, and therefore, several studies have attempted to improve the likability of robots by modifying their appearances and behavior [27,28]. The application of the halo effect by touching robots will be another effective design technique to improve the likability of robots.

Second, the Capability personality impression was mainly enhanced by imparting resilient touch sensation to the participants. However, the Capability personality impression was 
Fig. 6 Comparisons of factor scores related to each personality impression factor among the forearms. Each error bar shows the standard error of the mean. $\dagger p<0.1, * p<0.05$, $* * p<0.01, * * * p<0.001$. a Likability factor, $\mathbf{b}$ capability factor, c vitality factor

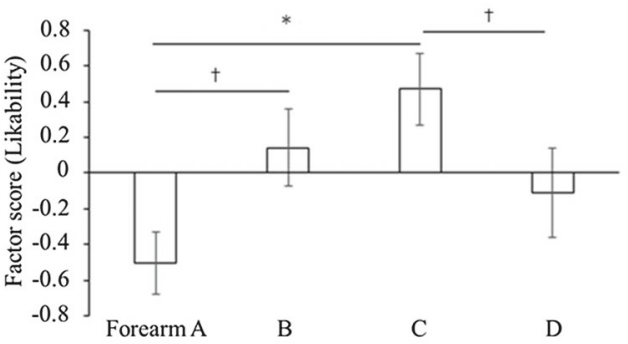

(a) Likability factor

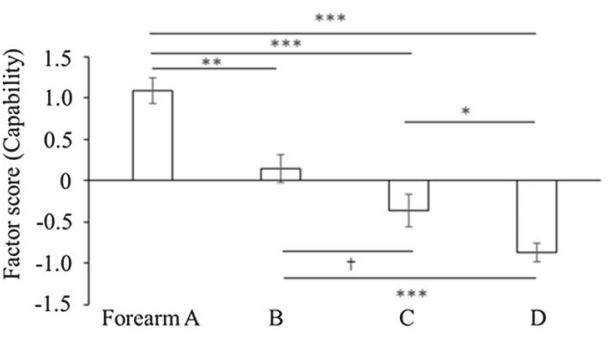

(b) Capability factor

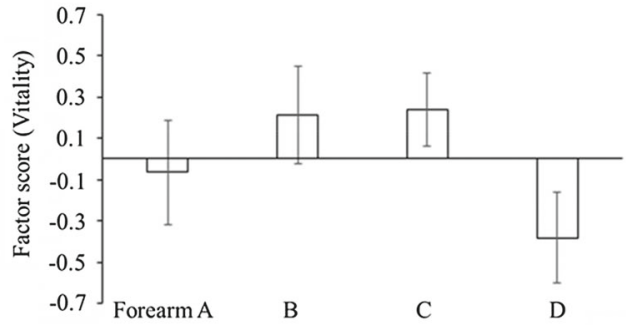

(c) Vitality factor

reduced by smooth and natural touch impressions. This suggests that supple, tight, rigid, and hard covering materials with rough, coarse, and dry surfaces and sharp and squared shapes are desirable to impart the Capability personality impression to robots. From this perspective, hard covering materials such as metal, plastic, and hard rubber are considered to be desirable to enhance the Capability personality impression of robots.

Third, the Vitality personality impression was enhanced by three of the four touch sensation factors: Preference, Resilience, and Naturalness. These results are unexpected but valuable findings especially for social robot designers.

Here, we encounter a new research question: whether both Likability and Capability personality impressions can be improved using the same covering materials. When we cover robots with soft and comfortable materials, the Likability personality impression will be improved, whereas the Capability personality impression will be degraded. On the other hand, when we cover robots with hard and unnatural materials, the Capability personality impression will be improved, whereas the Likability personality impression will be degraded. To improve both the personality impressions, covering materials that can provide both preferable and resilient touch sensations simultaneously to humans should be identified.

We can identify several issues for future consideration from the ANOVA results. First, the relationship between a robot's appearance and a human's preference for forearms should be investigated. In this study, forearms A and D, which were evaluated to be the most or least resilient, respectively (Fig. 5b), failed to help the robot to provide the Likability personality impression to the participants (Fig. 6a). The possible reason for this is that these forearms gave the participants a sense of incongruence in terms of the forearms of the child-type android robot because their surface elasticities measured by the durometer were stronger than those of the co-contracted forearms of males or weaker than those of the relaxed forearms of females. However, we could not conclusively determine whether this result was universally applicable to different types of robots. We should investigate how the factor scores related to the Preferable factor change when these forearms are attached to different types of robots such as a non-android robot.

Second, additional experiments are necessary to confirm whether the viscosity of the forearms affect the touch sensations and therefore the personality impressions. We prepared four types of forearms having different amounts of two types of additives that changed the elasticity and viscosity of these forearms. However, in this experiment, the order of scores in terms of the Resilience factor (Fig. 5b) was similar to the order of surface elasticities reduced by using both the additives, and therefore, we could not conclude that the difference in viscosity affected the evaluation by the participants. To make such a conclusion, we should use forearms with different viscosities and the same elasticity in a future experiment.

Third, we should be careful while considering the mutual relationships among touch sensation factors. Although the four types of forearms had cylinders of the same size and were covered with the same thin film, their Smoothness and Naturalness touch sensations were different: Forearm A, which had the highest elasticity, was evaluated to be rougher and coarser (Fig. 5c), whereas Forearm D, which had the lowest elasticity, was evaluated to be the moistest and most rounded. Robot designers should have sufficient knowledge about such human touch sensation properties; otherwise, it would be difficult to design touch sensations for effective personality impression design of robots. 


\section{Conclusion}

In this study, factor analysis, path analysis, and ANOVA were carried out on the evaluation scores of a robot's touch sensations and personality impressions by using SD questionnaires to show how the touch sensations affect the personality impressions (e.g., in the case of halo effect). Several significant causal relationships between touch sensations and personality impressions were found. The study results are expected to help in the design of the personality impressions of robots by facilitating a more systematic design of touch sensations. Further studies are required to reveal the types of covering materials that will provide preferable touch sensations to humans when using several types of robots, including non-android robots.

\section{Compliance with Ethical Standards}

Conflict of interest The authors declare that they have no conflict of interest.

Informed consent All participants provided written informed consent approved by the Ethics Committee of Graduate School of Engineering, Osaka University.

Open Access This article is distributed under the terms of the Creative Commons Attribution 4.0 International License (http://creativecomm ons.org/licenses/by/4.0/), which permits unrestricted use, distribution, and reproduction in any medium, provided you give appropriate credit to the original author(s) and the source, provide a link to the Creative Commons license, and indicate if changes were made.

\section{References}

1. Ekman P (1965) Communication through nonverbal behavior: a source of information about an interpersonal relationship. In: Affect, cognition and personality, pp 390-442

2. Argall BD, Billard AG (2010) A survey of tactile human-robot interactions. Robot Auton Syst 58(10):1159-1176

3. Macdorman KF (2006) Subjective ratings of robot video clips for human likeness, familiarity, and eeriness: an exploration of the uncanny valley. In: Iccs/cogsci-2006 long symposium: toward social mechanisms of android science

4. Bartneck C, Kanda T, Ishiguro H, Hagita N (2007) Is the uncanny valley an uncanny cliff? In: The 16th IEEE international symposium on robot and human interactive communication, pp 368-373

5. Kanda T, Miyashita T, Osada T, Haikawa Y, Ishiguro H (2008) Analysis of humanoid appearances in human-robot interaction. IEEE Trans Robot 24(3):725-735

6. Castro-Gonzlez A, Admoni H, Scassellati B (2016) Effects of form and motion on judgments of social robots' animacy, likability, trustworthiness and unpleasantness. Int J Hum Comput Stud 90:27-38

7. Scherer K (2003) Vocal communication of emotion: a review of research paradigms. Speech Commun 40(1-2):227-256

8. Yamashita Y, Ishihara H, Ikeda T, Asada M (2016) Path analysis for the halo effect of touch sensations of robots. In: Proceedings of the 8th international conference of social robotics, pp 502-512
9. Cramer H, Kemper NA, Amin A, Evers V (2009) The effects of robot touch and proactive behaviour on perceptions of humanrobot interactions. In: International conference on human robot interaction, pp 275-276

10. Cramer BH, Kemper N, Amin A, Wielinga B, Evers V (2009) 'Give me a hug': the effects of touch and autonomy on people's responses to embodied social agents. Comput Anim Virtual Worlds 20:437-445

11. Yohanan S, MacLean KE (2012) The role of affective touch in human-robot interaction: human intent and expectations in touching the haptic creature. Int J Soc Rob 4(2):163-180

12. Cooney MD, Nishio S, Ishiguro H (2015) Importance of touch for conveying affection in a multimodal interaction with a small humanoid robot. Int J Hum Rob 12(01): 1550002

13. Kozima H, Michalowski MP, Nakagawa C (2008) Keepon: a playful robot for research, therapy, and entertainment. Int J Soc Rob 1(1):3-18

14. Lee JK, Stiehl WD, Toscano RL, Breazeal C (2009) Semiautonomous robot Avatar as a medium for family communication and education. Adv Rob 23(14):1925-1949

15. Shibata T, Wada K (2011) Robot therapy: a new approach for mental healthcare of the elderly - a mini-review. Gerontology 57(4):378-86

16. Kanoh M, Shimizu T (2011) Developing a robot Babyloid that cannot do anything. J Rob Soc Jpn 29(3):298-305 (in Japanese)

17. Minato T, Nishio S, Ishiguro $H$ (2013) Evoking affection for a communication partner by a robotic communication medium. In: Hri demonstration session, pp D07

18. Shirado H, Nonomura Y, Maeno T (2007) Development of artificial skin having human skin-like texture. Trans Jpn Soc Mech Eng Ser C 73(726):541-546

19. Mori Y, Saito Y, Kamide H (2012) Evaluation of impression for hug dolls. J Jpn Soc Kansei Eng 11(1):9-15

20. Endo N, Iida F, Endo K, Mizoguchi Y, Zecca M, Takanishi A (2010) Development of the anthropomorphic soft robotic hand WSH-1R. In: Proceedings of the first iftomm asian conference on mechanism and machine science, pp 250162

21. Ishihara H, Asada M (2015) Design of 22-DOF pneumatically actuated upper body for child android 'Affetto'. Adv Rob 29(18):11511163

22. Osgood CE (1952) The nature and measurement of meaning. Psychol Bull 49(3):197-237

23. Inoue M, Kobayashi T (1985) The research domain and scale construction of adjective-pairs in a semantic differential method in Japan. Jpn J Educ Psychol 33(3):253-260 arXiv:1011.1669v3

24. The R Foundation (2016) The R project for statistical computing 8/1(January):1-3. Available from: https://www.r-project.org/. Accessed 1 Oct 2015

25. Minato T, Yoshikawa Y, Noda T, Ikemoto S, Ishiguro H, Asada M (2007) CB2: a child robot with biomimetic body for cognitive developmental robotics. In: Proceedings of international conference on humanoid robots, pp 557-562

26. Nisbett RE, Wilson TD (1977) The halo effect: evidence for unconscious alteration of judgments. J Personal Soc Psychol 35(4):250-256

27. Goetz J, Kiesler S, Powers A (2003) Matching robot appearance and behavior to tasks to improve human-robot cooperation. In: Proceedings of the IEEE international workshop on robot and human interactive communication, pp 55-60

28. Poel M, Heylen D, Nijholt A, Meulemans M, Breemen A (2009) Gaze behaviour, believability, likability and the iCat. AI Soc 24(1):61-73 
Publisher's Note Springer Nature remains neutral with regard to jurisdictional claims in published maps and institutional affiliations.

Yuki Yamashita received the Bachelor's degree in Engineering from the School of Engineering, Osaka University in 2016. He received the Master's degree in Engineering from the Department of Adaptive Machine Systems, Graduate school of Engineering, Osaka University in 2018. His research interests include effects of social touch in human-robot interaction.

Hisashi Ishihara is Tenure Track Assistant Professor at the Graduate School of Engineering, Osaka University since 2014. He received the Bachelor and Master's degrees in Engineering from Osaka University in 2007 and 2009, respectively. He also received the Ph.D. degree in Engineering from Osaka University in 2014. Since 2016, he has been a JST SAKIGAKE/PRESTO researcher. He is a visiting researcher of the laboratory for language development in RIKEN brain science institute and a collaborating researcher in Advanced Telecommunications Research Institute International. His research interests include human friendly robot body system, bio-inspired robotics, soft robotics, affective caregiver-child interactions, and cognitive developmental robotics.

Takashi Ikeda is an assistant professor at the Research Center for Child Mental Development, Kanazawa University since 2016. He received the Bachelor and Master's degrees at Kyoto University in 2003 and 2005, respectively. He also received the Ph.D. at Graduate school of Letters, Kyoto University in 2008. He worked as a specially appointed assistant professor at Osaka University from 2009 to 2016. His main research interest is on cognitive neuroscience.

Minoru Asada received B.E., M.E., and Ph.D. degrees in control engineering from Osaka University, Osaka, Japan, in 1977, 1979, and 1982, respectively. In April 1995, he became a Professor at Osaka University. Since April 1997, he has been a Professor in the Department of Adaptive Machine Systems at the Graduate School of Engineering, Osaka University. Dr. Asada has received many awards such as the Best Paper award at the IEEE/RSJ International Conference on Intelli- gent Robots and Systems (IROS92) and a Commendation by the Minister of Education, Culture, Sports, Science and Technology, Japanese Government, as a Person of Distinguished Services to Enlightening People on Science and Technology. He is one of the founders of the RoboCup, and the former president of the International RoboCup Federation (2002-2008). He was the Research Director of the ASADA Synergistic Intelligence Project at Exploratory Research for Advanced Technology by the Japan Science and Technology Agency (ERATO, 2005-2011), and was a principal investigator of the Grants-in-Aid for Scientific Research (Research Project Number: 24000012, 20122016) titled Constructive Developmental Science based on Understanding the Process from Neurodynamics to Social Interaction. $\mathrm{He}$ is currently a principal investigator of the JST RISTEX R\&D Project titled Legal Beings: Electiric personhoods of artificial intelligence and robots in NAJIMI society, based on a reconsideration of the concept of autonomy. 